\title{
ÜBERSICHT ÜBER DIE IM INVENTAR BERÜCKSICHTIGTEN ARCHIVE
}

\author{
Bundesarchiv und andere Archive mit Zuständigkeit für das ganze \\ ehemalige Reichsgebiet
}

\section{Bundesarchiv (BA)}

Für die schriftliche Überlieferung der zentralen Reichsbehörden, soweit sie sich nicht im Auswärtigen Amt, Politisches Archiv, befinden, und Dienststellen der NSDAP und für Schriftgut aus eingegliederten und besetzten Gebieten ist künftig ausschließlich das - Bundesarchiv, Abteilungen Potsdam (BA P)

Berliner Str. 98-101, 14467 Potsdam zuständig, in das mit Ausnahme der Bestände der Ostdokumentation und der Zeitgeschichtlichen Sammlungen und Nachlässe nach und nach alle entsprechenden Bestände aus Koblenz (BA KO, Potsdamer Str. 1, 56075 Koblenz) überführt werden und dem das bisherige Berlin Document Center als

- Außenstelle Berlin-Zehlendorf (BDC)

Wasserkäfersteig 1, 14163 Berlin, angeschlossen ist. Die aus dem Ministerium für Staatssicherheit der DDR und dem ehemaligen Zentralen Parteiarchiv der SED sowie der Dokumentationsstelle der DDRArchivverwaltung übernommenen und sonstige Unterlagen vornehmlich von Gerichten und Polizeidienststellen werden einstweilen im

- Bundesarchiv - Zwischenarchiv (BA DH)

Lindenallee 55-57, 15366 Dahlwitz-Hoppegarten, aufbewahrt, die Wehrmachtakten des ehemaligen Militärarchivs der DDR im

- Bundesarchiv, Abteilung Militärarchiv (BA-MA)

Wiesentalstr. 10, 79115 Freiburg, wehrmachtgerichtliche und Personalunterlagen weiterhin bei der

- Bundesarchiv - Zentralnachweissstelle (ZNS)

Abteigarten 6, 52076 Aachen (Kornelimünster), die Bestände des ehemaligen Staatlichen Filmarchivs der DDR gehören zum

- Bundesarchiv, Abteilung Filmarchiv

Fehrbelliner Platz 3, 10707 Berlin.

Die Benutzung ist geregelt durch die Verordnung über die Benutzung von Archivgut beim Bundesarchiv (BArchBV) vom 29. Oktober 1993 (BGBI. I S. 1857).

Lit: Vgl. Band I, S. 531, ferner D. MUREGGER, F. WINKLER: Quellen zur Geschichte der deutschen Arbeiterbewegung im „NS-Archiv“ des ehemaligen Ministeriums für Staatssicherheit (MFS) der DDR. 1994. - The HOLDINGS of the Berlin Document Center. 1994.

Schriftgut aus den Konzentrationslagern ist weiterhin beim

Internationalen Suchdienst (ISD)

Große Allee 5-9, 34444 Arolsen,

für wissenschaftliche Forschungen nicht zugänglich. 
Lit.: INTERNATIONALES KOMITEE vom Roten Kreuz, Internationaler Suchdienst: Inventar: Konzentrationslager und Historische Abteilung. 1957.

Institut für Zeitgeschichte (IfZ)

Leonrodstr. 46b, 80636 München

Deutsche Bibliothek - Deutsche Bücherei (DBL)

Deutscher Platz 1, 04103 Leipzig

\section{Staatsarchive der früheren und heutigen Länder}

\section{BERLIN}

\section{Landesarchiv Berlin (B)}

Kalckreuthstr. 1-2, 10777 Berlin

mit Außenstelle (ehem. Stadtarchiv Ost-Berlin)

Breite Str. 30-31, 10178 Berlin

Lit.: R. LIENING u. a.: Das Stadtarchiv Berlin. 1973. - Das LANDESARCHIV Berlin und seine Bestände. 2. Aufl. 1992.

\section{BRANDENBURG}

Die Benutzung ist geregelt durch Gesetz über die Sicherung und Nutzung von öffentlichem Archivgut im Land Brandenburg vom 7. April 1994 (GVBl. I S. 94).

\section{Brandenburgisches Landeshauptarchiv (P)}

Sanssouci-Orangerie, 14469 Potsdam

Zuständig für Überlieferung der Behörden der Provinz Mark Brandenburg und staatliche Behörden für die Reichshauptstadt und der entsprechenden Reichsmittelbehörden und Dienststellen der NSDAP.

Lit.: ÜBERSICHT über die Bestände des Brandenburgischen Landeshauptarchivs Potsdam. Teil II. 1967. ARCHIVALISCHE Quellennachweise zur Geschichte der deutschen Arbeiterbewegung Bd. 3. 1963. - SPEZIALINVENTAR zur Geschichte der bürgerlichen Parteien und Verbände in Deutschland bis 1945. Potsdam 1967. - BRANDENBURGISCHE ARCHIVE. Mitteilungen aus dem Archivwesen des Landes Brandenburg. $1993 \mathrm{ff}$.

\section{MECKLENBURG-VORPOMMERN}

\section{Landeshauptarchiv Schwerin (SN)}

Graf-Schack-Allee 2, 19053 Schwerin

Zuständig für Überlieferung der Behörden des Landes Mecklenburg und von Reichsmittelbehörden sowie Dienststellen der NSDAP in Mecklenburg.

\section{Landesarchiv Greifswald (GW)}

Martin-Andersen-Nexö-Platz 1, 17489 Greifswald

Zuständig für Überlieferung von Behörden der Provinz Pommern und entsprechender Dienststellen der NSDAP, soweit sie sich nicht im Archiwum Panstwowe Szczeczin befinden. 
Lit.: ARCHIVALISCHE Quellennachweise zur Geschichte der deutschen Arbeiterbewegung Bd. 7. 1962.

\section{PREUSSEN}

Geheimes Staatsarchiv Preußischer Kulturbesitz (GStA)

Archivstr. 12-14, 14195 Berlin (Dahlem)

Zuständig für Überlieferung der preußischen Zentralbehörden einschließlich der früher im Zentralen Staatsarchiv der DDR, Historische Abteilung II, in Merseburg aufbewahrten Bestände und für in das Gebiet der Bundesrepublik gelangtes Schriftgut von Behörden aus Ostbrandenburg, Pommern, Schlesien und Ostpreußen.

Lit.: ÜBERSICHT über die Bestände. Teil I. 1966. - K. FORSTREUTER: Das Preußische Staatsarchiv in Königsberg [jetzt im Geheimen Staatsarchiv]. 1955.

\section{SACHSEN}

\section{Sächsisches Hauptstaatsarchiv (DD)}

Archivstr. 14, 01097 Dresden, mit

- Außenstelle Bautzen, Seidauer Str. 2, 02625 Bautzen, und

- Außenstelle Glauchau (bis Ende 1994)

Zuständig für Überlieferung sächsischer Zentralbehörden und sächsischer sowie Reichsmittelbehörden und entsprechender Dienststellen der NSDAP.

\section{Sächsisches Staatsarchiv Leipzig (L)}

Beethovenstr. 4, 04107 Leipzig

Zuständig für Unterbehörden aus der Kreishauptmannschaft Leipzig, jedoch auch mit Beständen von Mittelbehörden.

Lit.: R. GROSS: Das staatliche Archivwesen in Sachsen. 1993. - ders.: Das Sächsische Hauptstaatsarchiv. 1994. - ÜBERSICHT über die Bestände des Sächsischen Landeshauptarchivs und seiner Landesarchive. 1955. - Sächsisches Archivblatt. Mitteilungen aus dem sächsischen Archivwesen. 1992 ff. - ARCHIVALISCHE Quellennachweise zur Geschichte der deutschen Arbeiterbewegung Bd. 5. - M. UNGER u. a.: Sächsisches Staatsarchiv Leipzig. Bestandsverzeichnis. 1992. - M. KÜLOW: Sächsisches Staatsarchiv Leipzig. Archivalische Quellennachweise zum Einsatz von ausländischen Zwangsarbeitern sowie Kriegsgefangenen während des Zweiten Weltkriegs. Zwangsarbeiterinventar. (1992). - S. HELD u. a.: Sächsisches Staatsarchiv Leipzig. Archivalische Quellennachweise zur Geschichte der Juden. Judaica-Inventar. 1993.

\section{SACHSEN-ANHALT}

\section{Landeshauptarchiv Sachsen-Anhalt (MD)}

Hegelstr. 25, 39104 Magdeburg

Zuständig für Überlieferung von Provinzialbehörden der preußischen Provinz Sachsen, von Reichsmittel- und preußischen Behörden in den Regierungsbezirken Magdeburg und Merseburg und entsprechender NSDAP-Dienststellen in den Gauen Magdeburg-Anhalt und Halle-Merseburg, mit

- Außenstelle Oranienbaum (OBA), Schloß, 06785 Oranienbaum, zuständig für Überlieferung der Behörden des Landes Anhalt, und

- Außenstelle Wernigerode, Lindenallee 21, 38855 Wernigerode

Lit:: GESAMTÜBERSICHT über die Bestände des Landeshauptarchivs Magdeburg. 1954-1960. - ARCHI-

VALISCHE Quellennachweise zur Geschichte der deutschen Arbeiterbewegung Bd. 4. 1963. - H. ROSS:

Entwicklung, Aufbau und Aufgaben des Landesarchivs Oranienbaum. 1963. 


\section{THÜRINGEN}

Die Benutzung ist geregelt durch Thüringer Gesetz über die Sicherung und Nutzung von Archivgut (ThürArchivG) vom 23. April 1992 (GuVOBl, S.139).

\section{Thüringisches Hauptstaatsarchiv Weimar (WE)}

\section{Marstallstr. 2, 99423 Weimar}

Zuständig für Überlieferung der Zentralbehörden des Landes Thüringen und für nachgeordnete und Reichsbehörden und der NSDAP, soweit nicht anderen thüringischen Archiven zugewiesen.

Lit.: ARCHIVFÜHRER Thüringen 1993. - Archive in Thüringen. Mitteilungsblatt. 1992 ff. - ÜBERSICHT über die Bestände des Thüringischen Landeshauptarchivs Weimar. 1959. - ARCHIVALISCHE Quellennachweise zur Geschichte der deutschen Arbeiterbewegung Bd. 6. 1963. - KURZE ÜBERSICHT über die Bestände des Thüringischen Hauptstantsarchivs Weimar. 1992.

- Außenstelle Gotha (GTH), Schloß Friedenstein, 99867 Gotha

Zuständig für preußische Behörden aus dem Regierungsbezirk Erfurt und thüringische aus den Kreisen Eisenach, Gotha und Sondershausen.

Lit.: ÜBERSICHT über die Bestände des Landesarchivs Gotha. 1960. - ARCHIVALISCHE Quellennachweise zur Geschichte der deutschen Arbeiterbewegung Bd. 6. 1963.

\section{Thüringisches Staatsarchiv Altenburg (ABG)}

Schloß 2a, 04600 Altenburg

Zuständig für nachgeordnete thüringische Behörden im Stadt- und Landkreis Altenburg.

Lit.: ÜBERSICHT über die Bestände des Landesarchivs Altenburg. 1961. - ARCHIVALISCHE Quellennachweise zur Geschichte der deutschen Arbeiterbewegung Bd. 6. 1963.

\section{Thüringisches Staatsarchiv Gotha (GTH)}

Schloß Friedenstein, 99867 Gotha

Zuständig für preußische Behörden aus dem Regierungsbezirk Erfurt und thüringische aus den Kreisen Eisenach, Gotha und Sondershausen.

Lit.: ÜBERSICHT über die Bestände des Landesarchivs Gotha. 1960. - ARCHIVALISCHE Quellennachweise zur Geschichte der deutschen Arbeiterbewegung Bd. 6. 1963.

\section{Thüringisches Staatsarchiv Greiz (GRZ)}

Oberes Schloß, 07973 Greiz

Zuständig für nachgeordnete thüringische und Reichsbehörden in den Kreisen Gera, Greiz und Schleiz.

Lit.: ÜBERSICHT über die Bestände des Landesarchivs Greiz. 1963. - Kurze Übersicht über die Bestände der Außenstelle Greiz. 1993.

\section{Thüringisches Staatsarchiv Meiningen (MGN)}

Schloß Bibrabau, 98617 Meiningen

Zuständig für nachgeordnete thüringische Behörden in den Kreisen Meiningen, Hildburghausen und Sonneberg und für Reichsbehörden in Thüringen.

Lit.: ÜBERSICHT über die Bestände des Staatsarchivs Meiningen. 1960. - ARCHIVALISCHE Quellennachweise zur Geschichte der deutschen Arbeiterbewegung Bd. 6. 1963. - KURZE ÜBERSICHT über die Bestände des Thüringischen Staatsarchivs Meiningen. 1991. 
Thüringisches Staatsarchiv Rudolstadt (RU)

Schloß Heidecksburg, 07407 Rudolstadt

Zuständig für nachgeordnete thüringische Behörden und Reichsbehörden in den Kreisen

Arnstadt, Rudolstadt, Saalfeld und Sondershausen.

Lit:: ÜBERSICHT über die Bestände des Landesarchivs Rudolstadt. 1964. - ARCHIVALISCHE Quellennachweise zur Geschichte der deutschen Arbeiterbewegung Bd. 6. 1963. - KURZE ÜBERSICHT über die Bestände des Thüringischen Staatsarchivs Rudolstadt. 1992.

\section{Universitäts- und Hochschularchive}

Humboldt-Universität zu Berlin, Universitätsarchiv (HUB), August-Bebel-Platz, 10117 Berlin

Technische Universität Dresden, Universitätsarchiv, Mommsenstr. 13, 01069 Dresden

Lit.: M. LIENERT: Das Universitätsarchiv Dresden. 1994.

Bergakademie Freiberg, Archiv, Akademiestr. 22, 09599 Freiberg

Ernst-Moritz-Arndt-Universität Greifswald, Universitätsarchiv, Domstr. 11, 17489

Greifswald

Martin-Luther-Universität Halle-Wittenberg, Universitätsarchiv, Weidenplan 12 , 06108 Halle

Friedrich-Schiller-Universität Jena, Universitätsarchiv, Goetheallee 1, 07743 Jena

Universität Leipzig, Universitätsarchiv, Beethovenstr. 6, 04107 Leipzig

Universität Rostock, Universitätsarchiv, Universitätsplatz 1, 18055 Rostock

\section{Sonstige Aufbewahrungsstellen}

Archiv der Reichsbahndirektion Berlin, Schöneberger Ufer 1-2, 10785 Berlin

Archiv der Reichsbahndirektion Halle, Ernst-Kamieth-Str. 2, 06112 Halle

KZ-Gedenkstätte Buchenwald, 99427 Weimar-Buchenwald

KZ-Gedenkstätte Mittelbau-Dora, 99734 Nordhausen

Mahn- und Gedenkstätte Langenstein-Zwieberge, 38805 Halberstadt

\section{Archive und Aufbewahrungsstellen im Ausland}

\section{ÖSTERREICH}

\section{Österreichisches Staatsarchiv}

- Archiv der Republik (AdR), Nottendorfer Gasse 2, A-1030 Wien

Zuständig für die Überlieferung aller für ganz Österreich zuständigen Reichs- und Landesbehörden und NSDAP-Dienststellen sowie der Wehrmacht, soweit nicht als Nachakten älterer Behörden in den Abteilungen

- Haus-, Hof- und Staatsarchiv (HHStA), Minoritenplatz 1, A-1010 Wien, oder

- Allgemeines Verwaltungsarchiv (AVA), Nottendorfer Gasse 2, A-1030 Wien

Lit.: Das ÖSTERREICHISCHE STAATSARCHIV. 1988 (Benutzungsbestimmungen dort S. 15. - A. STAUDINGER: Materialien in den Abteilungen des Österreichischen Staatsarchivs zum Thema „Wien und der AnschluB“". 1978. - ARCHIV der Republik. Eine Bestandsübersicht. 1992. 
Landesarchive

Kärntner Landesarchiv (KLA)

Landhaus, A-9020 Klagenfurt

Niederösterreichisches Landesarchiv (NÖLAW)

Herrengasse 11, A-1014 Wien

Oberösterreichisches Landesarchiv (LZ)

Anzengruberstr. 19, A-4020 Linz

Salzburger Landesarchiv (SZ)

Michael-Pacher-Str. 14, A-5010 Salzburg

Lit.: F. KOLLER: Das Salzburger Landesarchiv. 1987.

Steiermärkisches Landesarchiv (GR)

Bürgergasse 2a, A-8010 Graz

Lit.: GESAMTINVENTAR des Steiermärkischen Landesarchivs. 1959.

Tiroler Landesarchiv (IN)

Herrengasse 1, A-6010 Innsbruck

Vorarlberger Landesarchiv (BRG)

Kirchstr. 28, A-6900 Bregenz

Lit.: K. H. BURMEISTER: Das Vorarlberger Landesarchiv. 1967.

Wiener Stadt- und Landesarchiv (StLAW)

Rathaus, Felderstr. 1, A-1082 Wien

Lit.: P. CSENDES: Das Wiener Stadt- und Landesarchiv. 1991.

Sonstige Archive, Universitäts- und Hochschularchive

Dokumentationsarchiv des Österreichischen Widerstandes (DÖW)

Wipplinger Str. (Altes Rathaus), A-1010 Wien

Lit.: DOKUMENTATIONSARCHIV des österreichischen Widerstandes. 1982. - KATALOGE (der Archivbestände). 1963-1975.

Archiv der Karl-Franzens-Universität Graz, Universitätsplatz 3, A-8010 Graz

Archiv der Leopold-Franzens-Universität Innsbruck, Innrain 52,

A-6020 Innsbruck

Archiv der Universität Wien, Postgasse 9, A-1010 Wien

Universitätsarchiv der Technischen Universität Wien, Karlsplatz 13, A-1040 Wien

Direktion der Wirtschaftsuniversität Wien (ehem. Hochschule für Welthandel), Augasse 2-6, A-1090 Wien

Direktion der Universität für Bodenkultur, Gregor-Mendel-Str. 33, A-1180 Wien 
Direktion der Veterinärmedizinischen Hochschule Wien, Linke Bahngasse 13, A1030 Wien

Konsistorialarchiv Salzburg, Kapitelplatz 2, A-5010 Salzburg

\section{POLEN}

Staatliche Archive

Archivgut deutscher und preußischer Behörden aus Pommern befindet sich in den Archiven in Stettin, Köslin und Stolp, aus Schlesien in Breslau, Grünberg, Oppeln und Kattowitz, aus Ostpreußen in Allenstein und Elbing und den zugehörigen Zweigstellen, aus den 1939 annektierten Gebieten in Bromberg, Danzig, Posen, Lodz, Thorn, Kattowitz und Warschau.

Archiwum Panstwowe w Bydgoszczy (Staatsarchiv Bromberg, BRO) Dworcowa 65, PL-85-009 Bydgoscz

Archiwum Panstwowe w Gdansku (Staatsarchiv Danzig, DAN) Waly Piastowskie 5, PL-80-958 Gdansk

Lit:: C. BIERNAT: Archiwum Panstwowe w Gdansku (mit deutscher Zusammenfassung). 1992.

Archiwum Panstwowe w Elblagu (Staatsarchiv Elbing, ELB)

Zamek, PL-82-200 Malbork

Archiwum Panstwowe w Katowicach (Staatsarchiv Kattowitz, KAT) Jagiellonska 25, PL-40-950 Katowice, mit

- Filia w Gliwicach (Zweigarchiv Gleiwitz), ul. Zygmunta Starego 8, PL-44-100 Gliwice

Lit:: A. GROBELNY: Prameny $k$ dejinam Ceskoslovenska za nazisticke okupace v Statnim archivu w Katovicich. 1966.

Archiwum Panstwowe w Koszalinie (Staatsarchiv Köslin, KÖ)

Zwyciestwa 117, PL-75-950 Koszalin

Archiwum Panstwowe w Lodzi (Staatsarchiv Lodz, LO)

Plac Wolnosci, PL-90-950 Lodz

Lit.: M. CYGANSKI: Archiwalia z czasow okupacji Hitlerowskiej WAPM Lodzi i Wojewodzwa Lodzkiego. 1958.

\section{Archiwum Panstwowe w Olsztynie (Staatsarchiv Allenstein, ALL)}

Zamkowa 3, PL-10-074 Olsztyn

Lit.: K. CYBULSKA: Zasob wojewodzkiego Archiwum Panstwowegu w Olsztynie. 1982. - T. GRYGIER: Akta Rejencji Olsztynskiej. 1962.

Archiwum Panstwowe w Opolu (Staatsarchiv Oppeln, OP)

Zamkowa 2, PL-45-951 Opole, mit

- Filia Brzegu (Zweigarchiv Brieg), ul. Chrobrego 17, PL-49-301 Brzeg

Lit.: S. CZECH, R. DERMIN: Informator o zasobie archywalnym . . w Opolu. 1977. 
Archiwum Panstwowe w Poznaniu (Staatsarchiv Posen, POS)

ul. 23 Lutego 41, PL-61-967 Poznan

Lit.: C. SKOPOWSKI: Archiwum Panstwowe Miasta Poznania i Wojewodztwa Poznanskiego. 1969.

Archiwum Panstwowe w Slupsku (Staatsarchiv Stolp)

ul. Buczka 17, PL-76-200 Slupsk

Archiwum Panstwowe w Szczecinie (Staatsarchiv Stettin, STE)

Wojciecha 13, PL-70-410 Szczecin

Lit.: H. LASINSKI: Wojewodzkie Archiwum Panstwowe w Szczenie Przewodnik. 1964.

Archiwum Panstwowe w Toruniu (Staatsarchiv Thorn, THO)

Pl. Rapackiego 4, PL-87-100 Torun

Archiwum Panstwowe w m.st. Warszawy (Staatsarchiv der Hauptstadt Warschau, WA)

Krzywe Kolo 7, PL-00-270 Warszawa

Lit.: INFORMATOR: Archiwum Panstwowe w m.st. Warszawy. 1992.

Archiwum Panstwowe w Wroclawiu (Staatsarchiv Breslau, BRE)

ul. Pomorska 2, PL-50-215 Wroclaw, mit

- Filia w Legnicy (Zweigarchiv Liegnitz), ul. Piastowska 22, PL-59-220 Legnica und

- Filia w Walbrzychu (Zweigarchiv Waldenburg), ul. Poniatowskiego 57, PL-58-370

Boguszow

Lit.: INFORMATOR dla korzystajazych z materialow archiwalnych. 1976.

Archiwum Panstwowe w Ziloney Gorze (Staatsarchiv Grünberg, GRU)

Pionierow Lubuskich 53, PL-66-002 Stary Kiesilin - Zielona Gora

Centralne Archiwum Wojskowe (Zentrales Militärarchiv, MAW)

PL-00-910 Warszawa-Rembertow

Lit:: B. WOSZYNSKI: Das Zentrale Militärarchiv in Warschau und seine Bestände. 1969.

Panstwowe Muzeum w Oswiecimin (Staatliches Museum Auschwitz, PMO)

Blok 1, PL-32-605 Oswiecim 5

Panstwowe Muzeum w Sztutowe (Staatliches Museum Stutthof, MS)

PL-82-110 Sztutowo

Archiwum Glownej Komisji Badania Zbrodni Przeciwko Narodowi Polskiemu, Instytut Pamieci Narodowej (Archiv der Kommission zur Aufklärung von Verbrechen am polnischen Volk, Nationale Gedenkstätte, GKW)

Krakowskie Przedmiescie 25, PL-00-953 Warszawa 
Hochschularchive und wissenschaftliche Institute

Archiwum Uniwersytetu Wroclawskiego (Archiv der Universität Breslau), Plac Uniwersytecki 1, PL-50-137 Wroclaw

Archiwum Uniwersytetu im. Adama Mickiewicza (Archiv der Adam-MickiewiczUniversität Posen), H. Wieniawskiego 1, PL-61-712 Poznan

Instytut Zachodni (Westinstitut, IZ), Stary Rynek 2, PL-61-967 Poznan

Archiwum zydowskiego Instytutu Historycznego (Archiv des jüdischen historischen Instituts), Tlomackie 3/5, PL-00-090 Warszawa

\section{TSCHECHISCHE REPUBLIK}

Státní ústrední archiv v Praze (Staatliches Zentralarchiv in Prag)

Karmelitská 2, CZ-118 01 Praha 1 - Malá Strana

Zuständig für Schriftgut ziviler Zentralbehörden und zentraler Einrichtungen der NSDAP im ehem. Protektorat Böhmen und Mähren.

Moravsky zemsky archiv v Brno (Mährisches Landesarchiv Brünn, BRN)

Zerotinovo nam. 3/5, p.p.1, CZ-662-02 Brno

Státní oblastní archiv v Litomericích (Staatliches Gebietsarchiv Leitmeritz, LT)

Krajská 1, CZ-412 74 Litomerice

Státní oblastní archiv v Plzni (Staatliches Gebietsarchiv Pilsen, PZ)

Sedláckova 44, p.p. 312, CZ-306 12 Plzen

Státní oblastní archiv v Treboni (Staatliches Gebietsarchiv Trebon)

Zamek, CZ-379 11 Trebon

Státní okresní archiv v Decíne (Staatliches Kreisarchiv Tetschen)

Zizkova 4, CZ-405 02 Decín IV

Zemsky archiv v Opave (Landesarchiv Troppau, TRO)

Snemovní 1, CZ-746 22 Opava

RUSSLAND

Zentrum für die Aufbewahrung historisch dokumentarischer Sammlungen, Vyborskaja ul. 3, Moskau

Lit.: G. ALY, S. HEIM: Das Zentrale Staatsarchiv in Moskau („Sonderarchiv“. 1992. - B. WEGNER: Deutsche Aktenbestände im Moskauer Zentralen Staatsarchiv. 1992. - K. JENA, W. LENZ: Die deutschen Bestände im Sonderarchiv in Moskau. 1992. 
